Meta

Journal des tradlucteurs

Translators' Journal

\title{
L'échec du Faulkner comique en France : un problème de réception
}

\section{Annick Chapdelaine}

Volume 34, numéro 2, juin 1989

URI : https://id.erudit.org/iderudit/002217ar

DOI : https://doi.org/10.7202/002217ar

Aller au sommaire du numéro

Éditeur(s)

Les Presses de l'Université de Montréal

ISSN

0026-0452 (imprimé)

1492-1421 (numérique)

Découvrir la revue

Citer cet article

Chapdelaine, A. (1989). L'échec du Faulkner comique en France : un problème

de réception. Meta, 34(2), 268-279. https://doi.org/10.7202/002217ar d'utilisation que vous pouvez consulter en ligne.

https://apropos.erudit.org/fr/usagers/politique-dutilisation/ 


\section{L'ÉCHEC DU FAULKNER COMIQUE EN FRANCE* : UN PROBLEME DE RECEPTION}

ANNICK CHAPDELAINE

Université McGill, Montréal, Canada

\section{I - INTRODUCTION}

À la lumière de l'historique de la réception française de Faulkner et de son «impossible» [sic] réhabilitation comique, nous postulons que l'échec du Faulkner comique en France est essentiellement un problème de réception. Réception du texte de départ par les traducteurs qui n'y ont pas vu ni lu de comique, réception renforcée par des critiques importants, dont l'un est traducteur de Faulkner, et ensuite, réception des traductions par les lecteurs français qui n'étaient pas ou plutôt plus en mesure de lire du comique où il n'avait pas été inscrit, c'est-à-dire dans le texte d'arrivée.

\section{II - HISTORIQUE DE LA RÉCEPTION FRANÇAISE DE FAULKNER}

Les premières traductions de romans, au nombre de cinq, publiées en France entre 1933 et 1938, produits des années merveilleuses de la création de Faulkner, sont les suivantes: Sanctuaire (1933), Tandis que j'agonise (1934), Lumière d'août (1935), Sartoris (1937) et Le Bruit et la fureur (1938). Malheureusement, elles ne sortirent pas dans le même ordre de publication que les textes américains puisque, outre Soldier's Pay (1926) et Mosquitoes (1927), ces cinq romans parurent aux États-Unis dans l'ordre suivant: Sartoris (1929), The Sound and the Fury (1929), As I Lay Dying (1930), Sanctuary (1931), Light in August (1932). La chronologie des traductions de Faulkner en France constitue déjà un premier handicap pour la réception du comique puisque Mosquitoes, tout en étant d'une moins grande teneur que les œuvres qui lui ont succédé, a été vite reconnu comme un roman à portée humoristique.

Or, dès son lancement en France, surtout avec la parution de Sanctuaire, roman «noir» par excellence, Faulkner a été acclamé comme un des plus grands écrivains tragiques de son temps. Et ses premiers «hérauts» en France ne furent autre que l'un de ses principaux traducteurs, Maurice-Edgar Coindreau, qui a traduit trois sur cinq des romans cités ci-dessus (Tandis que j'agonise, Lumière d'août et Le Bruit et la fureur) et en a préfacé deux (Lumière d'août et Le Bruit et la fureur), précédé de deux préfaciers on ne peut plus célèbres, André Malraux pour Sanctuaire et Valery Larbaud pour Tandis que j'agonise.

Aucune de ces préfaces, fait remarquable, n'aborde les possibilités comiques de l'œuvre, sauf peut-être Malraux qui les effleure en écrivant ceci: "cc'est l'absurdité qui donne à ses personnages secondaires, presque comiques (la maitresse du bordel avec ses chiens), une intensité comparable à celle de Chtchédrine», ou encore Maurice-Edgar Coindreau, qui, dans sa préface du Bruit et la fureur les renie en ces termes: «Telle est la composition de cette symphonie démoniaque où ne manque que la gaieté d'un scher$z o$ \%. Remarquons qu'il ne faut pas assimiler gaieté à humour et comique et, par consé- 
quent, en employant le terme «gaieté», Coindreau avait raison. Car ce qui est drôle, nous en conviendrons, n'est pas forcément gai.

Sinon, en reprenant les préfaces une à une, on ne lit que: «Monde où l'homme n'existe qu'écrasé», «Et peut-être l'irrémédiable est-il son seul vrai sujet», «le sentiment qui fait la valeur de l'cuure de Faulkner : la haine», «l'art tragique» chez André Malraux pour Sanctuaire; "Et tout l'épisode, à partir du moment où Addie rend le dernier soupir, $n$ 'est que le récit de ces étranges et interminables obsèques», "Et à côté de la transposition en Epique, l'imagination du lecteur se trouve assez sollicitée pour inventer une transposition en dramatique» de Valery Larbaud pour Tandis que j'agonise; "cette fresque sauvage et passionnée», "ces ouvrages mystérieux où les scènes les plus atroces se voilent d'obscurités perfides», "Enfoui dans l'irrémédiable, comme l'a si justement écrit André Malraux [déjà Malraux est repris], il [Faulkner] possède au plus haut degré ce «sentiment tragique de la vie» qu'analysa Unanumo. La Fatalité enveloppe un monde où il ne voit qu' atrocités, où les hommes et les femmes, marionnettes aveugles du Destin, pièces d'échecs dans les mains du Joueur suprême, ne connaissent que le sang, la volupté et la mort», et le «Puritanisme» de Faulkner longuement développé et qui a fait école: «Puritanisme. Source de fanatisme et, par suite, la meilleure école de la haine, cette haine toujours latente dans les ouvrages de William Faulkner et qui déborde de toutes les pages de Lumière d'aô̂t", au point d'y assimiler le personnage de Lena Grove, pourtant source d'épisodes comiques et pastoraux, à «des Thaïs promenant sous leur fard la plus vile lubricité : Lena Grove et Milly, si pressées de se laisser séduire», et puis viennent les termes de «vomissement», «Fatalité», «la haine», "objet d'horreur», "sa conception de la réalité et du temps», «un technicien habile», "un grand artiste», qui sont presque devenus des clichés sur Faulkner, et en dernier lieu:

S'il lui arrive de faire dresser les cheveux sur la tête, ... c'est à la façon des artistes du moyen âge qui, courbés sous le joug de l'Église et dominés par l'idée du péché, peignaient des danses macabres, sculptaient des figures de cauchemar et imaginaient, pour humilier la chair, des accouplements monstrueux,

fin de la préface de Maurice-Edgar Coindreau à Lumière d'août. Rappelons que Coindreau a traduit ce roman. «M. Faulkner se contente d'ouvrir les portes de l'Enfer», «Jason est un monstre de fourberie et de sadisme», «Son oncle Jason la poursuit de sa haine, et c'est là vraiment tout le sujet du livre, la haine de Jason au cours des journées du 6, 7, et 8 avril 1928», affirme-t-il. Certes, mais ajoutons, traité sur le mode comique. Phrases suivies d'une analyse des procédés d'écriture de Faulkner «pour faciliter au lecteur l'accès de cette œuvre complexe», la lecture de William Faulkner étant «une petite science», clôturée par «la beauté tragique», à nouveau par Maurice-Edgar Coindreau pour Le Bruit et la fureur.

Il est frappant de constater l'univocité «tragique» des préfaciers, à tel point que Coindreau ne voit en Lena Grove et Jason Compson que des personnages maléfiques traités sur le mode tragique, alors que la première est une simple et sereine fille-mère, et le second, horrible, certes, mais tous deux, de façon différente, sources d'épisodes comiques. Par ailleurs, et nous tenons à le dire, ces préfaciers ont tous fait preuve d'intuitions remarquables. André Malraux a prononcé un mot-clé sur l'œuvre de Faulkner : «la fascination», Valery Larbaud a vu le caractère épique de Tandis que j'agonise, et Maurice-Edgar Coindreau a bien senti la dimension tragique, immense et réelle, de l'œuvre de Faulkner. Mais il reste qu'aucun d'entre eux n'en a perçu la dimension comique.

Il convient cependant d'expliquer que chacun a inscrit sa lecture dans sa préface. Il serait d'ailleurs intéressant, comme Derycke 1'a fait pour Malraux, de faire des rappro- 
chements entre le romancier et ses préfaciers. Ainsi l'écrivain Malraux s'est attaché à «la signification éthique de l'événement qui importe davantage que l'événement lui-même» (Derycke 1935: 697), Valery Larbaud a fait ouvre de critique en ouvrant des portes d'interprétation de Tandis que j'agonise, et Maurice-Edgar Coindreau, à la fois traducteur et professeur de français à Princeton pendant trente-huit ans (1923-1961), a joué un rôle exemplaire de pédagogue et d'initiateur de Faulkner pour les lecteurs français, tenant à démontrer qu'il ne fallait pas s'arrêter devant les aspects choquants ou techniquement ardus de l'écrivain, mais les surmonter pour y lire une œuvre qui les transcende. C'est d'ailleurs à lui que revient la «découverte» de Faulkner ainsi que la publication du premier article français consacré à cet auteur - et le troisième dans le monde - paru en juin 1931 dans la Nouvelle Revue Française. Dans cet article, il initie les Français au talent de Faulkner, déclarant, sans hésitation, que ce dernier est «actuellement une des figures les plus intéressantes de la jeune littérature américaine» (Coindreau 1931: 926). Le mérite de Coindreau est grand puisque c'est grâce à son remarquable jugement littéraire qu'il a «trouvé» Faulkner et l'a traduit pour son pays. Les effets de son entreprise, il faut le dire et le redire, ont d'une part permis à Faulkner d'être connu en France et d'autre part enrichi la scène littéraire française de l'époque, et ce pour toujours.

Hormis la consécration de Faulkner par ses préfaciers, un autre grand écrivain et critique de Faulkner en France, Jean-Paul Sartre, saisi lui aussi par la découverte du grand romancier américain, en cristallise à son tour, et pour longtemps, l'image dans son pays. En février 1938, il publie dans la Nouvelle Revue Française, un article intitulé «Sartoris, par W. Faulkner», et en juillet 1939, toujours dans la N.R.F., une analyse du Bruit et la fureur, "A Propos du Bruit et la Fureur: la temporalité chez Faulkner". Dans le premier, on lit: «J'ai compris le grand ressort de son art: la déloyauté», «les gestes, c'est le monde de l' ennui», "Le véritable drame est derrière, derrière l' ennui, derrière les gestes, derrière les consciences», et, il finit sur : «Il faudrait le connaître». Dans le second, comme l'indique son sous-titre, «la temporalité chez Faulkner», Sartre analyse la métaphysique de Faulkner, qu'il dénomme «une métaphysique du temps». Citons : «Le malheur de l'homme est d'être temporel ... Tel est le véritable sujet du roman», «l'enfoncement», «l' irrationalité du temps», «L' homme passe sa vie à lutter contre le temps et le temps ronge l'homme comme un acide, l'arrache à lui-même et l'empêche de réaliser l' humain. Tout est absurde...»; "L'homme de Faulkner, créature privée de possibles et qui s'explique seulement par ce qu'il était, vous ne le reconnaîtrez pas en vous-même». Et enfin, une explication qui révèle comme pour Malraux, écrivain lui aussi, que Sartre était trop engagé dans son œuvre propre pour se distancer de celle de Faulkner: "pour lui, comme pour nous tous, l'avenir est barré». Mais c'est dans son exposition sur la «Situation de l'écrivain en 1947» que Sartre met vraiment le doigt sur ce qui a fait le succès de Faulkner et des écrivains américains pour leurs homologues français :

...ce n'est pas par leur cruauté ni par leur pessimisme qu'ils nous ont touchés: nous avons reconnu en eux des hommes débordés, perdus dans un continent trop grand comme nous l'étions dans l'histoire et qui tentaient, sans traditions [sic], avec les moyens du bord, de rendre leur stupeur et leur délaissement au milieu d'événements incompréhensibles. ... Nous ne définirions plus, je crois, la beauté par la forme ni même par la matière, mais par la densité d'être. (Sartre $1948: 255-256$ )

À nouveau, pas un mot sur l'art comique, qui s'est vu, une fois de plus, gommé au profit de l'art tragique.

Il est important de préciser que la réception et la critique américaines de William Faulkner n'ont pas non plus réservé au comique le traitement qu'il méritait, ni au tragique 
d'ailleurs, du moins au début, comme cela avait été immédiatement le cas en France. Il serait par contre injuste de souscrire entièrement aux déclarations de Maurice-Edgar Coindreau qui, dans plusieurs articles, déplore le manque de reconnaissance que Faulkner a reçu de ses concitoyens, plus frappés par la violence, la folie et la décadence de ses écrits, ainsi que par ses difficultés de style. Car aux États-Unis, comme en France, des écrivains et des artistes, dont Conrad Aiken, Warren Beck, Katherine Anne Porter, Robert Penn Warren et Eudora Welty ont vite reconnu en Faulkner un maître. Comme l'explique Thomas L. McHaney dans sa préface à William Faulkner : A Reference Guide, la différence entre la divulgation du talent de Faulkner dans son pays et en France réside, en partie, dans le fait que les intellectuels américains ne possédaient pas un organe de diffusion de la portée de la N.R.F. Ainsi, "American writers who championed Faulkner did not reach as wide a readership as their European counterparts and moreover had to compete with a basically hostile press of large and loud proportions» (McHaney 1976: x). Il est certain que les thèmes scandaleux de Faulkner heurtaient beaucoup plus les sensibilités nationales que celles de l'étranger. Seulement, en revenant au seul comique qui nous intéresse ici, si l'on ne cite que ces écrivains américains, il faudra attendre 1946 pour que Robert Penn Warren, en développant les thèmes de Faulkner aux yeux de la critique américaine, parle de l'importance de "l' humour, surtout l' humour frontalier» dans l'ouvre du grand écrivain (Warren 1946: 176-80). Puis, Katherine Anne Porter, en 1948, écrit: "William Faulkner has the deepest and most serious humor in this country at present» (Porter 1948: 17)). Eudora Welty, à son tour, en 1949, dans un article sur Intruder in the Dust, célèbre l'humour qui ressort des situations terribles du roman (Welty 1949: 59698). Et enfin, Warren Beck, dans Man in Motion : Faulkner's Trilogy (1961), mais là il s'agit d'une analyse de la trilogie des Snopes, dont le premier volume a paru en 1940, s'est montré très sensible au comique chez Faulkner.

Néanmoins, le seul recueil d'articles consacré uniquement à l'humour chez Faulkner ne paraîtra que sur l'initiative des éditrices Doreen Fowler et Ann J. Abadie. La première déclarant dans son introduction: «William Faulkner, the recognized genius of tragic art, is a master of comic forms as well and, further, [..] neither mode, tragic or comic, is ever very far from the other in Faulkner's world» (Fowler et Abadie 1986: ix). Ces critiques organisèrent un colloque portant le titre approprié de Faulkner and Humor à l'Université du Mississippi en ... 1984. La publication qui en découla, traite, entre autres, des premières cuvres que nous abordons ici: Jason dans Le Bruit et la fureur («Humor as Vision in Faulkner», James M. Cox; «Jason Compson: A Demoralized Wit», William N. Claxon, Jr.), Tandis que j'agonise («The Comic World of As I Lay Dying», Patricia R. Schroeder), Lumière d'août ("The Levity of Light in August», Virginia V. Hlavsa), 1'importance du «tall tale» dans l'œuvre de Faulkner ( What Faulkner Learned from the Tall Tale», Thomas L. McHaney), la réception du comique et de l'humour en Allemagne ( Faulkner's Humor: A European View», Hans Bungert) où Faulkner semble avoir passé l'épreuve de l'étranger sans trop de difficultés, contrairement aux États-Unis où, selon Bungert: «Scholars were somewhat slow in taking up the challenge presented by remarks that Malcolm Cowley and Robert Penn Warren made about Faulkner's humor in 1946», mais d'ajouter "but by now a considerable, though still not sufficiently large, amount of scholarship exists on the subject» (Fowler et Abadie : 140-41), puis l'emploi des bandes dessinées chez Faulkner, dont est en partie issu le personnage de Popeye dans Sanctuaire ( Faulkner Reads the Funny Papers», M. Thomas Inge). S'ensuit une analyse psychanalytique de l'humour de Faulkner dans Soldier's Pay, Sartoris, The Sound and the Fury, As I Lay Dying et surtout Sanctuary («Lacan and Faulkner: A PostFreudian Analysis of Humor in the Fiction», James M. Mellard). Et enfin, dans «Fix My Hair, Jack»: The Dark Side of Faulkner's Jokes», titre qui fait allusion aux dernières 
paroles du violeur à l'épi de maïs, Popeye, sur l'échafaud, l'écrivain George Garrett ferme le recueil sur ces mots :

a great man [Faulkner] whose vision was so open and inclusive that if he always felt the tears of things, he likewise could hear, as some hear voices, the sourceless laughter which is at least half the music of this world. (Fowler et Abadie : 230-31)

III - LA RÉHABILITATION DU FAULKNER COMIQUE EN FRANCE : UNE MISSION IMPOSSIBLE ?

Comme nous venons de le constater, la réhabilitation du Faulkner comique a été et est possible aux États-Unis. A quoi l'attribuer? A la re-lecture, aux nouvelles lectures, du texte original de Faulkner, du système-texte, non déformé par l'opération traduisante. La situation est tout autre pour les versions françaises des romans de Faulkner. A quoi l'attribuer? D'une part à l'orientation des traducteurs qui ont produit un texte prisonnier de leur propre lecture tragique, d'autre part, à la confirmation de cette optique par la consécration des critiques presque trop célèbres qui ont, bien malgré eux sans doute, figé une vision unidimensionnellement tragique de l'œuvre du grand romancier.

Or Sartre ne lisait pas l'anglais (Gresset 1977: 1355, note 1), et nous ne savons toujours pas si Malraux le faisait. Ainsi, lorsque dans sa préface, Malraux écrit: «ll n'y a pas $d$ " «homme» de Faulkner, ni de valeurs, ni même de psychologie, malgré les monologues intérieurs de ses premiers livres», tout donne lieu de croire que Malraux avait lu dans le texte The Sound and the Fury ou As I Lay Dying, puisqu'en 1933 aucun roman de Faulkner n'avait encore paru en traduction française. Ou alors, comme Michel Gresset pose bien la question: "Ou bien avait-il seulement lu l'article publié par Maurice-Edgar Coindreau en 1931? Il n'est pas indifférent de savoir si les commentateurs d'importance lurent Faulkner dans l'original ou en traduction» (Gresset 1977: 1355, note 1). De ce côté donc, la question reste entière. Valery Larbaud, pour sa part, semble avoir lu As I Lay Dying en anglais comme le démontre sa correspondance (Gresset 1966:26-28) avec Coindreau lorsqu'il en rédigeait la préface, quoiqu'il ait exprimé, à plusieurs reprises, les difficultés que le texte original lui causait, particulièrement sur le plan de la langue vernaculaire. Ces difficultés permettent de croire d'une part que certains aspects de l'œuvre, le comique n'étant pas le moindre, ont pu lui échapper, et que d'autre part, il a dû s'appuyer sur la traduction de Tandis que j'agonise pour en faire la préface. C'est donc, en définitive, aux traductions mêmes qu'il faut se reporter. Celles de René-Noël Raimbault et Henri Delgove pour Sanctuaire et Sartoris, et celles de Maurice-Edgar Coindreau pour Tandis que j'agonise, Lumière d'août et Le Bruit et la fureur. L'heure est donc venue de «relire» les traductions de Faulkner en français pour en saisir la lecture non-comique.

\section{IV - DE LANNEXION DU COMIQUE A SON DÉCENTREMENT}

Pourtant, les cinq premiers romans de Faulkner traduits en France, et qui plus est, coup sur coup, ont chacun une dimension comique. Faut-il le rappeler, l'œuvre de Faulkner, le grand œuvre, se distingue par le fait qu'il rassemble la pluralité des langues de son époque, ici américaines, du parler populaire (les Blancs pauvres, les Noirs) à la langue des romans épiques, des romans pastoraux et des œuvres littéraires plus grandes que nature qui ont recours aux mythes classiques. Et dans cet ouvre, ces langues s'entrelacent et s'ironisent mutuellement. Souvent à des fins tragiques, mais parfois aussi à des fins comiques. Le comique réside dans cet entrelacement même, qu'il soit fusionné comme dans Tandis que j'agonise, "In Faulkner's As I Lay Dying, this fusion is complete», comme le dit si bien Carson McCullers dans «The Russian Realists and Southern Literature» (McCullers 1941), ou juxtaposé et mis en contrepoint comme dans Sartoris, Le Bruit et la fureur, Sanctuaire et Lumière d'août. À chaque fois le lecteur est 
secoué, que ce soit d'horreur ou de rire, mais il reste que par ses choix, le texte de Faulkner signifie par le biais du comique et que cette part signifiante de son œuvre a été occultée dans les traductions françaises.

Que faire? Ce que Faulkner a fait avec le Sud. Comme l'écrit V.S. Pritchett à propos de la Chute dans l'œuvre du romancier in «That Time and that Wilderness»: «[Faulkner] got to work on the South with pick and shovel, slinging up the earth, quarrying the rock-..., He knew there had been a Fall and that it was historical» (V.S. Pritchett 1962: 405). Cette métaphore d'une chute historique pourrait s'appliquer à ce que l'on appellera la «traduction-introduction» du langage-système comique de Faulkner, concept que nous tirons de Meschonnic dans Pour la poétique II, où il postule que «Selon l' historicité du traduire, une traduction est traduction-introduction, avant que soit produit, s'il peut l'être, le moment d'une traduction-texte» (Meschonnic 1973: 313). Car, toujours selon Meschonnic:

La traduction-introduction ... se définit par le possible d'une époque. Le possible d'une époque est la somme de ses idées reçues. ... C'est pourquoi chaque époque retraduit. La traduction non-texte vieillit: étant passivement la production d'une idéologie, elle passe avec cette idéologie. ... Alors que le texte ne vieillit pas: il se transforme. Le possible d'une époque n'est pas une notion subjective (péjorative) seulement. C'est une notion subjectiveobjective, individuelle-collective. La somme des idées reçues désigne l'ensemble de ce qui peut être reçu (lu, écrit) et de ce qui ne peut pas être reçu. (Meschonnic 1973: 321)

Et c'est ce que nous proposons pour réhabiliter, ré-inscrire le comique dans l'œuvre de Faulkner en français: procéder à «l'écriture d'une lecture-écriture, aventure personnelle et non transparence, constitution d'un langage-système dans la langue-système tout comme ce qu' on appelle auvre originale» (Meschonnic 1973: 354). Or, comme le signale Meschonnic, une époque, une société, une classe produisent le traducteur pour un public. L'époque, pour les premières traductions de Faulkner, correspond à l'entre deuxguerres. La société, c'est l'institution littéraire française des années trente, gardienne de la culture française classique, d'une langue que, d'après Sartre: "cent cinquante ans de domination bourgeoise ont cassée, vulgarisée, assouplie, truffée de "bourgeoisismes" " (Sartre 1948: 203). Quant à la classe, il s'agit de la bourgeoisie, dont les écrivains possèdent tous le brevet: le baccalauréat; «en France où le baccalauréat est un brevet de bourgeoisie, il n'est pas admis qu'on projette d'écrire sans être au moins bachelier» (Sartre 1948: 204), exigence qui valait certainement aussi pour ses traducteurs. «On a les traductions qu'on mérite» (Meschonnic 1973: 358). Si, en effet, chaque époque, selon le moment historique et les rapports entre langues, transparaît dans ses traductions autant que dans ses œuvres, il n'est que de voir les œuvres marquantes de l'époque en France: La condition humaine, 1933, La Nausée, 1938 et L'Étranger, 1942, pour comprendre l'inévitable inscription «historique» du tragique dans les traductions de Faulkner en France.

Mais l'Amérique, les États-Unis, est un pays où la tradition littéraire est «Autre» que la française. Autre, parce que l'humour y a une place privilégiée, parce que c'est un mode d'écriture qui fait partie intégrante du tissu conjonctif même de la langue. Et Faulkner, héritier de cette tradition, l'a exploitée dans presque toutes ses cuvres. L'année de la publication de son premier roman, il en a parlé longuement dans une déclaration qu'il fit sur la relation entre l'humour et l'art où il citait l'humour comme un trait national louable:

We have one priceless universal trait, we Americans. That trait is our humor. What a pity it is that it is not more prevalent in our art. This characteristic alone, being national and indigenous, could, by concentrating our emotional forces inward upon themselves, do for us 
what England's insularity did for English art during the reign of Elizabeth. One trouble with us American artists is that we take our art and ourselves too seriously. And perhaps seeing ourselves in the eyes of our fellow artists, will enable those who have strayed to establish anew a sound contact with the fountainhead of our American life. (Faulkner 1926)

Faulkner, en ces mots, montrait qu'il ne s'écarterait pas, lui, du bon chemin de l'humour américain, qu'il se garderait bien de renier ses origines. Or, ses origines profondes plongent en grande partie leurs racines dans l'humour du sud-ouest américain caractérisé par le «tall tale», dans la tradition de Hooper, Tensas, Thorpe, Harris, Longstreet et Twain, tradition marquée par l'humour de l'exagération, une conception de la nature humaine dénuée de toute sentimentalité, et enfin une célébration des possibilités littéraires de la langue vernaculaire. En 1971, Hans Bungert a fondé sa thèse sur ce lien étroit qui existe entre Faulkner et l'humour du sud-ouest américain : William Faulkner und die humoristische Tradition des amerikanischen Südens, plus sensible peut-être qu'il était à cet élément de l'œuvre de Faulkner à cause de la tradition littéraire allemande. A telle enseigne que, dans son article intitulé «Faulkner's Humor: A European View», Bungert écrit qu'un critique allemand des Larrons a même appelé Faulkner «a genial Münchhausen» (Fowler et Abadie 1986: 140). Dans sa thèse donc, Bungert conclut que : «Unter seinen [de Faulkner] Romanen ist kaum einer, der nicht derartige in die humoristische Tradition zurückverfolgbare Züge aufwiese», à l'exception de Pylône qui, selon lui, est dépourvu d'humour (Bungert 1971: 177). Du côté français, Robert Escarpit, dans son petit livre sur l'humour, corrobore cette notion de l'humour essentiellement américain en disant:

Émigrant en Amérique, les Pères Pélerins avaient, à leur insu, emporté avec eux à bord du Mayflower les germes de l'humour. Ils mirent plus de deux siècles à percer jusqu'à la littérature, mais longtemps avant l'Indépendance un sense of humour (qui n'est d'ailleurs pas encore de l'humour) colore parfois le puritanisme de la Nouvelle-Angleterre. Il y a dans l'introspection puritaine, la recherche du péché caché, une conscience de soi qui, à la longue, est génératrice d' humour.

Un peu partout des formes d'humour très diverses naîtront en Amérique de situations variées : la tall story des frontières de l'Ouest, rodomontade épique qui n'est pas sans rapport avec le "gab" des preux de Charlemagne, l'impassible ironie des proverbes indiens, la bonhomie souriante du folklore noir, l'inlassable tendresse des Quakers et même le snobisme de l'humour qui fait que dans la société américaine plus encore que dans la société anglaise, avouer un manque de sense of humor (avec un o) est avouer une coupable infirmité. (Escarpit 1960: 57)

Faulkner, très tôt dans sa carrière d'écrivain, se flattait de posséder cette qualité : «mirth requires two things: humor and a sense of humor. I flatter myself that I possess the latter» (Faulkner 1920). Or, les traducteurs français ont mal perçu le véritable génie langagier dont Faulkner a fait montre dans ses écrits. Le fond inépuisable de l'humour du Sud et de la langue parlée américaine, où Faulkner s'est constamment ressourcé, semble leur avoir échappé. Stanley D. Woodworth, professeur de français aux États-Unis, qui dans William Faulkner en France (1931-1952) étudie l'accueil critique fait à Faulkner et son influence sur la littérature française, rapporte qu'il a vite senti le besoin d'analyser aussi les traductions de ses livres, à cause, précisément, "de l'écart significatif qui existe souvent entre l'original et la traduction» (Woodworth 1959: 6). Dans son chapitre I, intitulé «Problèmes de traduction», il relève deux problèmes particuliers de traduction: le style personnel de Faulkner, et son régionalisme. "Le sudisme de Faulkner, très complexe, très profond» est tel, selon lui, que «le Sud ne se traduit pas» (Woodworth 1959: 30-31). A l'aide d'exemples, il signale la difficulté de rendre le patois des noirs [sic] et 
celui des blancs [sic] et conclut que «Pratiquement, et avec raison, les traducteurs n'ont pas essayé de traduire le patois» (Woodworth 1959: 33). Pour le patois des Blancs, il s'appuie sur la conclusion de la préface de Valery Larbaud, qui ne peut que donner à réfléchir :

M. Maurice Coindreau, qu'on doit féliciter d'avoir mené à bien une tâche épineuse, a fait sagement en ne cherchant pas à rendre les caractéristiques du patois que parlent les personnages de Tandis que j'agonise. Ce patois peut être curieux pour le lecteur de langue anglaise (le verbe to aim y joue le rôle d'un auxiliaire), mais ce n'est guère qu' un anglais dégradé, entaché de négligence et de mauvaises habitudes, qui nous a paru plus difficile que savoureux, bien qu'il reflete les conditions d'existence de ceux qui le parlent et soit bien [c'est nous qui soulignons] dans l'atmosphère du livre.

Inutile d'insister sur la négation de la pure étrangeté de l'œuvre étrangère, sur la peur et le refus ethnocentrique face à ce que serait une traduction décentrée, ouverte, dialogique et métissée. Et c'est l'occultation de l'étranger dans l'cuvre de Faulkner qui aura mené à une occultation de sa dimension comique. Antoine Berman a longuement parlé de ce phénomène typiquement français en préconisant l'élargissement de la langue française pour en arriver à une traduction non-ethnocentrique. Il reproche à la littérature française classique son: "appauvrissement de la créativité orale, [sa] mort des dialectes, [son] enfoncement ... dans un espace de plus en plus clos où elle devient de moins en moins capable de «figurer» le monde» (Berman 1984: 288). Il faut, selon Berman, «mobiliser les ressources de notre langue pour la mettre à la hauteur de ces diverses ouvertures. ...une exigence maximale de «savoir» au service d'une certaine ré-alimentation de la capacité parlante du langage» (Berman 1984: 289). Et c'est en travaillant dans ce sens, en envisageant la traduction comme non annexion, mais décentrement, qu'elle deviendra «cette auvre double, ce dedans-dehors d'une langue et de sa littérature» (Meschonnic $1973: 360$ ) et qui est, en fait, sa véritable fonction. Elle est, comme le dit Meschonnic, «le grand emprunt» (Meschonnic 1973: 360).

Le gommage des sociolectes n'est pas la seule cause d'une lecture impossible du comique chez Faulkner en français. Il y est néanmoins pour une large part. C'est comme si le comique de Faulkner débordait les idiolectes de ses traducteurs, dont les procédés ont annexé, banalisé, aplani, et finalement effacé des passages, des structures, un texte enfin, pétris d'étranger et d'humour. Ce gommage de l'espace polylangagier d'une communauté aura réalisé en français une univocité de l'œuvre du grand écrivain, lui conférant pour seul statut celui de texte tragique, au lieu de produire une œuvre audacieuse dont la force et la richesse, la violence même, proviennent du mélange des deux genres, le comique et le tragique, faisant de Faulkner un auteur comique au même titre qu'un auteur tragique. Interrogé sur le rôle de l'humour dans son œuvre lors des conférences qu'il donna à l' Université de Virginie en 1957-1958:

$Q$. Sir, do you look at your humor as a - with the same inspiration as you do a serious thing or is it a more relaxing kind of work?.

Faulkner répondit :

A. No, no, it's a part of man too, it's a part of life. That people are - there's not too fine a distinction between humor and tragedy, that even tragedy is in a way walking a tightrope between the ridiculous - between the bizarre and the terrible. That it's - possibly the writer uses humor as a tool, that he's still trying to write about people, to write about man, about the human heart in some moving way, and so he uses whatever tool that he thinks will do most to finish the picture which at the moment he is trying to paint, of man. That he will use humor, tragedy, just as he uses violence. They are tools, but an ineradicable parts of life, that humor is. (Faulkner $1959: 39$ ) 
Pour rétablir la réputation de Faulkner auteur comique en France, il faudra donc procéder à une analyse des tendances déformantes qui opèrent dans une traduction, tendances qui aplatissent l'informité caractéristique de la grande prose. Berman, dans «la traduction et la lettre ou l'auberge du lointain», explique que dans la mesure où la prose est considérée comme inférieure à la poésie, les déformations y sont mieux acceptées quand elles ne passent pas inaperçues. Car elles portent souvent sur des points difficilement décelables! Berman ajoute, et ceci nous concerne directement: «Il est facile de voir en quoi un poème de Hölderlin a été massacré; il l' est moins de voir en quoi un roman de Faulkner l'a été, surtout si la traduction semble «bonne» (c'est-à-dire esthétique)» (Berman 1985: 68). S. D. Woodworth aura souscrit à cet état de fait pour les traductions françaises de Faulkner en écrivant, dans son chapitre sur les problèmes de traduction, que si l'on s'en tient à la réaction de la critique française, l'on n'a qu'à conclure que ces traductions sont toutes d'une égale qualité. D'autant plus que, presque sans exception, les comptes rendus des romans, s'ils parlent des traductions, le font dans des termes très élogieux. Et Woodworth de se poser la question clé sur les traductions de Faulkner: «On peut cependant se demander à quel point ces critiques sont vraiment capables de juger de la qualité d'une traduction», car, poursuit-il: «En allant de l'américain contemporain au français contemporain, il existe pour le pauvre traducteur d'énormes difficultés qui restent quelquefois sans solution" (Woodworth 1959: 18). Tout en étant d'accord sur 1'«incompétence» des critiques pour juger des textes traduits, et sur la difficulté, énorme, de rendre l'étrangeté américaine en français, nous ne pouvons nous rallier à Woodworth pour ce qui est de l'«impossibilité» de traduire Faulkner en français. Car cette impossibilité est historique et non pas définitive.

Antoine Berman touche à ce point très délicat de' la traduction, dans sa déclaration célèbre et souvent reprise, à savoir: «la traduction n' est pas une simple médiation : c'est un processus où se joue tout notre rapport avec l'Autre» (Berman 1984: 287). Il faut donc faire de la traduction une entreprise «excentrique» par un enrichissement de notre langue, un infléchissement de notre créativité littéraire pour ouvrir l'acte de traduire à un dialogue avec les autres cultures. Berman recommande d'analyser «les systèmes de déformation pesant sur toute opération de traduction» pour pouvoir "à partir de cette analyse, proposer un contre-système» (Berman 1984: 297). Or, sur les treize déformations de la traduction décrites par Berman (1), il en est une, l'«homogénéisation», regroupant d'ailleurs la majeure partie des tendances de ce système, qui touche de très près la problématique du gommage du comique chez Faulkner. Cette tendance, qui consiste à unifier sur tous les plans le tissu de l'original, aura réussi, dès Sanctuaire, pour ne citer que ce roman de Faulkner, le premier à paraître en France en 1933, à empêcher une lecture comique de plusieurs épisodes du livre qui le sont ouvertement, les faisant se fondre dans la masse tragique prédominante de l'œuvre au lieu de provoquer la rupture en contrepoint qui est leur fonction première.

Pour ne nommer que les plus évidents, il y a l'épisode des gars naîfs de la campagne, Virgil et Fonzo, dans le bordel qu'ils ne reconnaissent pas comme tel, le prenant pour le «sanctuaire» d'une grande famille ou encore d'une couturière avec beaucoup d'essayeuses (Sanctuaire, chapitre XXI). Cette mésaventure tire par ailleurs ses sources dans l'humour du sud-ouest américain ne serait-ce que par l'emploi du langage vernaculaire des deux compères. Ainsi, quand Virgil propose: "Let's git off this hyer street" (Sanctuary, 184) et que la traduction se lit «Prenons cette rue-là» (Sanctuaire, 184), ou encore, lorsqu'un peu plus loin, dépité d'avoir eu à débourser trois dollars pour une prostituée, il déclare: «Aint nothing worth three dollars you caint tote off with you» (Sanctuary, 190) traduit par « $Y$ a rien qui vaille trois dollars quand on peut pas l' emporter avec soi» (Sanctuaire, 189), on peut comprendre que le lecteur français ait du mal à 
percevoir les effets comiques à tirer du personnage. Il en est de même pour l'épisode de la veillée funèbre aux nappes noires de Red, non pas dans un salon funéraire mais dans une boîte de nuit aménagée pour l'occasion (ch. XXV), du «slapstick» américain de premier ordre, à renforts de coups de poings et de couronnes mortuaires. Et enfin, le «thé» des commères bien-pensantes, après les funérailles, toujours au chapitre $\mathrm{XXV}$, où, à grands renforts, cette fois-ci, de soupirs et d'alcool pour se "r'donner du coeur" (Sanctuaire, 248), ces dames, à mots couverts, s'indignent des pratiques sexuelles de Popeye :

Yes, sir, Minnie said the two of them would be nekkid as two snakes, and Popeye hanging over the foot of the bed without even his hat off, making a kind of whinnying sound. (Sanctuary, 251)

cette fois, quelque peu mieux traduit par:

Oui, Madame, Minnie m' disait qu'ils étaient tous deux nus comme des vers [Temple et Red, ce dernier invité par Popeye l'impuissant pour «servir» sa dame à sa place] et qu'Popeye, penché par-dessus le pied du lit, sans avoir même enlevé son chapeau, il faisait comme une espèce de hennissement. (Sanctuaire, 252)

Comment ne pas rire devant une scène pareille, et comment ne pas souscrire à une phrase que Faulkner a prononcée en 1920: «unless humor is, like evil, in the eye of the beholder» (Faulkner 1920). Baudelaire, dans son chapitre «De l'essence du rire» in Curiosités esthétiques; l'Art romantique et autres œuvres contemporaines, avait écrit, avant Faulkner: "Le comique, la puissance du rire est dans le rieur et nullement dans l'objet du rire» (Baudelaire 1962: 245). Au risque de voir du comique où il n'y en a pas, force est de constater que cette scène, pour «primitive», «perverse» ou «sordide» qu'elle soit, par la prise de conscience du ridicule de la situation humaine qu'elle opère, rappelle pour ainsi dire le lecteur à l'ordre au moyen d'un rire rectificatif.

Nous avons peut-être là, enfin, une explication de ce qui a causé le refus du Faulkner comique par les lecteurs, et par lecteurs, nous entendons les traducteurs, les préfaciers, les critiques et le grand public, français. Car si le sens étranger de l'œuvre n'avait pas été acclimaté, s'il n'y avait pas eu exclusion de bien des éléments linguistiques vernaculaires ou étrangers, détachement de la lettre du texte, de son corps mortel, de sa gangue terrestre, il n'y aurait pas eu la formidable annexion du comique, annexion au sens que lui prête Meschonnic dans ses «Propositions pour une poétique de la traduction»:

12. ...L'annexion est l' effacement de ce rapport [textuel], l'illusion du naturel, le comme-si, comme si un texte en langue de départ était écrit en langue d'arrivée, abstraction faite des différences de culture, d'époque, de structure linguistique.

par opposition au décentrement, qu'il définit ainsi:

12. Le décentrement est un rapport textuel entre deux textes dans deux langues-cultures jusque dans la structure linguistique de la langue, cette structure linguistique étant valeur dans le système du texte, (Meschonnic 1973: 308)

et vers lequel devrait tendre toute traduction.

$\mathrm{v}-$ CONCLUSION

Il ne faut pas avoir peur de croire que pour toute langue, on peut postuler une correspondance rigoureuse avec une autre langue, mais à un niveau «virtuel». Développer ces potentialités, comme le dit Berman, «telle est la tâche de la traduction, qui progresse donc vers la découverte de la "parenté» des langues», de là provient tout le sens de 
l'expression «transposition créatrice» (Berman 1984 : 303). Il faudrait en arriver au point où le texte d'arrivée aurait la même force créatrice que l'original, où la traduction d'une œuvre serait uniformisée en fonction des particularismes répétés de l'écrivain.

Pourquoi pas un travail qui s'inspirerait de celui qui se fait depuis 1978 au «Europäisches Übersetzer-Kollegium» à Straelen en R.F.A., dont Elmar Tophoven est le fondateur. Dans un article intitulé «Elmar Tophoven et le Collège européen des traducteurs», Annie Brisset expose la méthode élaborée par le professeur Tophoven: «Il commente ses traductions, qu'il fait sur ordinateur, les enregistrant avec leurs variantes préalables, leurs difficultés et les conclusions à en tirer pour les traductions à venir, les siennes ou celles des autres" (Brisset 1987: 8). La critique des traductions de Faulkner pourrait se faire dans cet esprit. Ce serait un travail collectif qui reconnaîtrait à la traduction son statut de champ pluridisciplinaire dans lequel les traducteurs pourraient fructueusement travailler avec les écrivains ou les exégètes de l'écrivain, les théoriciens de la littérature, les linguistes y compris les socio- et les ethnolinguistes, les lexicologues et les terminologues, et aussi, selon le cas, les psychanalystes et les philosophes.

C'est dans cette optique que nous proposons, à l'instar de Michel Gresset, et cinquante-cinq ans après la publication de Sanctuaire, "ni plus ni moins de "ré-américaniser» Faulkner» (Gresset 1985: 64), en cernant, une fois pour toutes, le travail sur «la lettre» inhérent à l'acte de traduire, pour rendre enfin justice et à Faulkner et à ses lecteurs de langue française.

Notes

* L'expression est de Michel Gresset («Aspects de la traduction littéraire : Poe et Baudelaire, Faulkner et ses interprètes», SSLM-Annuario, N. 1, Trieste, 1985, p. 65), grand faulknérien français qui, selon les paroles de l'un de ses homologues américains, Noel Polk : «[He] has established himself as among the premier critics of Faulkner writing in any language» (Michel Gresset, A Faulkner Chronology, translated from: Faulkner, Euvres Romanesques, by Arthur B. Scharff, foreword by Noel Polk, Jackson, University Press of Mississippi, 1985, p. ix).

1. «la rationalisation, la clarification, l'allongement, l'ennoblissement et la vulgarisation, Il'appauvrissement qualitatif, sic], l' appauvrissement quantitatif, l' homogénéisation, la destruction des rythmes, la destruction des réseaux signifiants sous-jacents, la destruction des systématismes textuels, la destruction lou l' exotisation) des réseaux langagiers vernaculaires, la destruction des locutions et idiotismes, l' effacement des superpositions de langues», in 3. «l'analytique de la traduction et la systématique de la déformation», «la traduction et la lettre ou l'auberge du lointain», les tours de babel, Mauvezin, Trans-Europ-Repress, 1985, pp. 68-69.

\section{BIBLIOGRAPHIE}

BAUDELAIRE, Charles (1962): Curiosités esthétiques; l'Art romanesque et autres auvres critiques, intro., notes et bibliographie par Henri Lemaire, Paris, Editions Garnier Frères.

BECK, Warren (1961): Man in Motion: Faulkner's Trilogy, Madison (Wisconsin), University of Wisconsin Press.

BERMAN, Antoine (1984) : L'Épreuve de l'étranger. Culture et traduction dans l'Allemagne romantique, Paris, Gallimard.

BERMAN, Antoine (1985) : «la traduction et la lettre ou l'auberge du lointain», les tours de babel : essais sur la traduction, Mauvezin, Trans-Europ-Repress.

BRISSET, Annie (juin 1987) : «Elmar Tophoven et le Collège européen des traducteurs», Circuit, $\mathrm{n}^{\circ} 17$, p. 8-10.

BUNGERT, Hans (1971): William Faulkner und die humoristische Tradition des amerikanischen Südens, Heidelberg, Carl Winter, Universitätsverlag.

COINDREAU, Maurice-Edgar (juin 1931): «William Faulkner», Nouvelle Revue Française, CCXXXVI, p. 926-30.

DERYCKE, Gaston (octobre 1935) : «Compte rendu de Sanctuaire», Cahiers du Sud, XXII, p. 697-98.

ESCARPIT, Robert (1960) : L'humour, Collection «Que sais-je ?», Paris, P.U.F. 
FAULKNER, William (1920): The Mississippian, 17 mars 1920, reproduit sous le titre «The Ivory Tower», dans William Faulkner: Early Prose and Poetry, édité par Carvel Collins, Boston, Toronto, Little, Brown and Company, 1969.

FAULKNER, William et Spratling, William (1926) : Sherwood Anderson and Other Famous Creoles, New Orleans, introduction (sans pagination).

FAUULKNER, William (1926) : Soldier's Pay, New York, Liveright Publishing Corporation, 1951.

FAULKNER, William (1927): Mosquitoes, New York, Liveright Publishing Corporation, 1951.

FAULKNER, William (1929) : Sartoris, New York, Random House, 1962.

FAULKNER, William (1937) : Sartoris, traduction de René-Noël Raimbault et Henri Delgove, Paris, Gallimard (Collection «Du Monde Entier»), 1949.

FAULKNER, William (1929) : The Sound and the Fury, New York, Random House, 1966.

FAULKNER, William (1938) : Le Bruit et la furewr, traduction et préface de Maurice-Edgar Coindreau, Paris, Gallimard (Collection «Du Monde Entier»), 1949.

FAULKNER, William (1930) : As I lay Dying, New York, Random House, 1964.

FAULKNER, William (1934): Tandis que j'agonise, traduction de Maurice-Edgar Coindreau, préface de Valery Larbaud, Paris, Gallimard (Collection «Du Monde Entier»), 1957.

FAULKNER, William (1931): Sanctuary, New York, Random House, 1958.

FAULKNER, William (1933): Sanctuaire, traduction de René-Noël Raimbault et Henri Delgove, préface d'André Malraux, Paris, Gallimard (Collection «Du Monde Entier»), 1949.

FAULKNER, William (1932): Light in August, New York, Random House, 1959.

FAULKNER, William (1935): Lumière d'août, traduction et préface de Maurice-Edgar Coindreau, Paris, Gallimard («Folio»), 1974.

FAULKNER, William (1959): Faulkner in the University, Class Conferences at the University of Virginia 1957-1958, edited by Frederick L. Gwynn and Joseph L. Blotner, Charlottesville, Virginia, The University of Virginia Press.

FOWLER, Doreen et ABADIE, Ann J. (éditrices, 1986) : Faulkner and Humor : Faulkner and Yoknapatawpha, 1984, Jackson and London, University Press of Mississippi.

GRESSET, Michel (juin 1966): «Valery Larbaud et les débuts de Faulkner en France», Preuves, ${ }^{\circ}{ }^{184}$, p. 26-28.

GRESSET, Michel (1977): Faulkner, CEuvres romanesques I, textes traduits par M.-E. Coindreau, H. Delgove, R.-N. Raimbault, revus par M. Gresset. Edition présentée et annotée par Michel Gresset, Paris, Gallimard (bibliothèque de la «Pléiade»).

GRESSET, Michel (1985a) : «Aspects de la traduction littéraire : Poe et Baudelaire, Faulkner et ses interprètes», SSLM-Annuario, N.1, Trieste, p. 60-81.

GRESSET, Michel (1985b) : A Faulkner Chronology, translated from: Faulkner, Euvres Romanesques, by Arthur B. Scharff, foreword by Noel Polk, Jackson, University Press of Mississippi.

MESCHONNIC, Henri (1973) : Pour la poétique II, Paris, Gallimard.

McCULLERS, Carson (juillet 1941): Decision, reproduit dans The Mortgaged Heart, Boston, Houghton Mifflin, 1971, p. 253-254.

McHANEY, Thomas L. (1976) : William Faulkner : A Reference Guide, G.K. Hall and Co., 70 Lincoln St., Boston, Mass.

PORTER, Katherine Anne (1948): Pageant of American Humor, letter to Edwin Seaver, quoted by Edwin Seaver, editor, Cleveland and New York, World Publishing Company.

PRITCHETT, V.S. (28 Septembre 1962): «That Time and That Wilderness», New Statesman, 405-406.

SARTRE, Jean-Paul (février 1938): «Sartoris, par W. Faulkner», Nouvelle Revue Française, L, p. 323-28. Repris dans Situations I, Paris, Gallimard, 1947, p. 7-13.

SARTRE, Jean-Paul (juin 1939) : «A Propos du Bruit et de la fureur : la temporalité chez Faulkner», N.R.F., LII, p. 1057-1061; LIII (juillet 1939), p. 147-151. Repris dans Situations I, Paris, Gallimard, 1947, p. 65-75.

SARTRE, Jean-Paul (1948) : «Situation de l'Écrivain en 1947», Situations II, Paris, Gallimard, p. 202-316.

WARREN, Robert Penn (1946): «Cowley's Faulkner», New Republic (12 August), p. 176-80; (26 August), p. 234-37.

WELTY, Eudora (1949) : «In Yoknapatawpha», Hudson Review, I (Winter), 596-98.

WOODWORTH, Stanley D. (1959): William Faulkner en France (1931-1952), Paris, M.J. Minard. 